\title{
Pichia cactophila, a New Species of Yeast Found in Decaying Tissue of Cacti
}

\author{
W. T. STARMER, $\dagger$ H. J. PHAFF, MARY MIRANDA, AND M. W. MILLER \\ Department of Food Science and Technology, University of California, Davis, California 95616
}

\begin{abstract}
A novel representative of the yeast genus Pichia has been recovered 190 times during the period 1971 to 1976 . We regard this organism as belonging to a new species, Pichia cactophila. Strains were found in the necrotic tissue of 16 species of cactus and in the crops of 3 species of Drosophila which utilize the cacti as host plants. Isolates were obtained from widely separate geographic localities (throughout Mexico, Southwestern United States, and Hawaii). The new species forms predominantly two-spored asci. Both homothallic and heterothallic strains were observed. The metabolic capability of $P$. cactophila, like that of $P$. membranaefaciens, is limited to oxidative utilization of only a few compounds. $P$. cactophila can be differentiated from $P$. membranaefaciens by its strong growth on D-glucosamine and by the lower guanine-plus-cytosine content ( 36.3 to 37.5 $\mathrm{mol} \%$ ) of its nuclear deoxyribonucleic acid. The type strain of $P$. cactophila is UCD-FST 76-243A (= ATCC 34932 = CBS 6926).
\end{abstract}

During a recent survey of yeasts associated with necroses of cacti of the North American Sonoran Desert $(4,14)$, we consistently isolated yeasts that were identified (5) as Pichia membranaefaciens Hansen. Subsequently we extended the range of our survey of the Sonoran Desert to include most of the peninsula of Baja California, Mexico. The resulting increase in the number of yeast isolates (ca. 500) added a large number of additional strains at first considered to belong to $P$. membranaefaciens. However, slight but consistent differences in the assimilation pattern of carbohydrates, correlated with host-plant specificity and determinations of the nuclear deoxyribonucleic acid (DNA) base compositions of representative strains, revealed that the isolates represented several homogeneous groups distinct from $P$. membranaefaciens. The guanine-plus-cytosine $(\mathrm{G}+\mathrm{C})$ contents of the DNAs of the different groups were found to be 8 to 16 percentage points lower than that of $P$. membranaefaciens. By the currently used identification procedures, however, these groups were not sufficiently distinct from the standard description of $P$. membranaefaciens to be considered separate species. Nevertheless, the intragroup homogeneity with respect to nuclear DNA base composition was suggestive of a new species. It was found that the new species, to be described in this paper, can be differentiated from $P$. membranaefaciens by its strong growth on D-glucosamine, which compound is not or is only weakly assimilated by the latter species.

\footnotetext{
$\dagger$ Present address: Department of Biology, Syracuse University, Syracuse, NY 13210.
}

We have named the new species $P$. cactophila (cac.to'phi.la. M. L. mas. n. Cactus generic narne of cactus; Gr. adj. philus loving; M. L. adj. cactophilus cactus-loving) on the basis of its frequent recovery from many species of cacti found in widely separated geographic areas. The other yeasts that we have recognized as new species will be described in subsequent reports.

\section{MATERIALS AND METHODS}

Samples of necrotic cactus tissue were collected in various areas of the North American Sonoran Desert (Fig. 1). A few samples were obtained from cacti in the Oaxaca area of Southern Mexico, and a single isolate came from a cactus on the island of Hawaii (Table 1). Most of the isolations were done by streaking a loopful of necrotic tissue directly on acidified yeast extractmalt extract agar (AYM; Difco YM agar plus $0.7 \%$ [vol/vol] $1 \mathrm{~N} \mathrm{HCl}, \mathrm{pH} 3.7$ to 3.8 ) in the field. In some cases dilutions of the tissue were made in sterile water as described earlier (14). The plates were stored at ambient temperatures $\left(24\right.$ to $\left.30^{\circ} \mathrm{C}\right)$ until colonies appeared. Counts of morphologically distinct colony types were then made, and a representative of each type was brought into pure culture by two successive platings on YM agar for identification. On the isolation medium, colonies of the new species were recognized with a fair degree of confidence, for they were flat, rather spreading, semiglossy to dull, and sometimes possessed a textured surface or a finely ciliate border.

Some isolates were obtained from adult drosophilae captured near cacti with necrotic tissue. The flies were surface-sterilized in $70 \%$ ethanol (for about $1 \mathrm{~min}$ ), rinsed in $10 \mathrm{ml}$ of sterile water, and then squashed and streaked directly on AYM agar plates in the field.

Identification was done by standard methods currently used in yeast taxonomy (16). DNA extraction and purification were done by a combination of the 


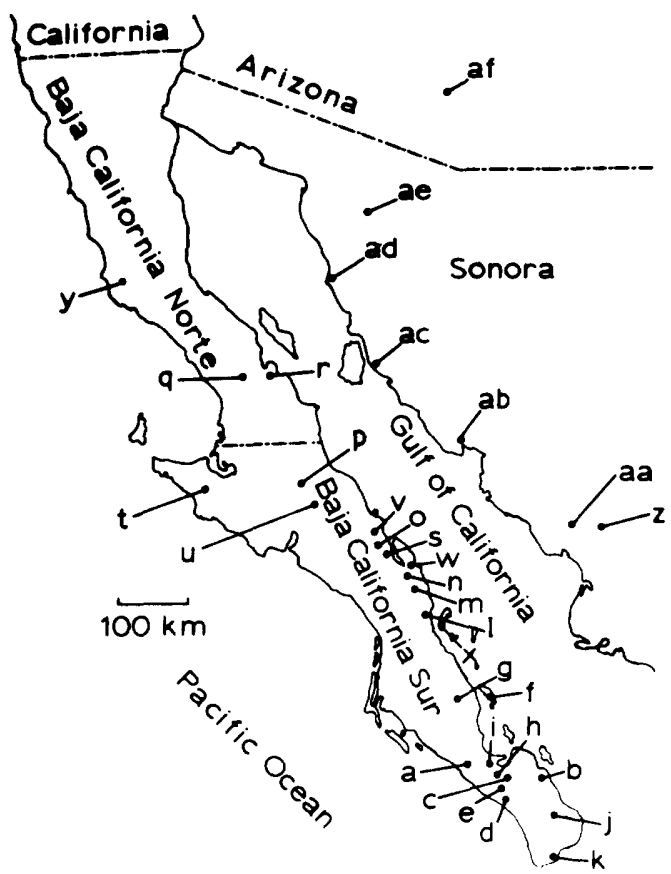

FIG. 1. Collection localities for isolates of Pichia cactophila listed in Table 1.

procedures of Marmur (7) and Bernardi et al. (1). The $\mathrm{G}+\mathrm{C}$ content (moles percent) of the DNA was calculated from buoyant density values in cesium chloride $(11,15)$ and was based on two to three separate determinations. Micrococcus lysodeikticus DNA, with a buoyant density of $1.7311 \mathrm{~g} / \mathrm{ml}$, was used as a reference. The buoyant density of the $M$. lysodeikticus DNA was derived from comparison with Escherichia coli K-12 DNA, whose buoyant density was taken to be 1.7100 .

Single ascospores were isolated from asci with a micromanipulator (3) after treatment of the sporulating suspension of cells and asci with the cell walldigesting enzyme zymolyase.

\section{RESULTS}

Latin diagnosis of the new species: Pichia cactophila sp. nov. In extracto malti cellulae ovoideae, $(1.8-5.7) \times(2.9-9.0) \mu \mathrm{m}$, singulae, binae, aut in catenis brevis; sedimentum, annulus et pellicula crispulata non-nitida formantur. Cultura in agaro malti post unum mensem $\left(20^{\circ} \mathrm{C}\right)$ cremea, glabra, semi-nitida, mollis; margine glabro, undulato, interdum vel pseudomycelium rudimentum. In agaro farinae Zea maïs post dies 10 , pseudomycelium nullum aut rudimentum.

Species homothallica aut heterothallica, diploidea. Asci inconjugati fiunt, habentes 2 sporos pileiformos (raro 4) in quoque asco; asci rumpunter.
Fermentatio glucosi nulla aut exigua. Glucosum, glucosaminum, ethanolum, glycerolum, acidum lacticum (variabile), acidum succinicum, et acidum citricum (raro nullum) assimilantur at non galactosum, L-sorbosum, maltosum, saccharum, cellobiosum, trehalosum, lactosum, melibiosum, raffinosum, melezitosum, inulinum, amylum solubile, D-xylosum, L-arabinosum, Darabinosum, D-ribosum, L-rhamnosum, methanolum, erythritolum, ribitolum, galactitolum, mannitolum, glucitolum, $\alpha$-methyl-D-glucosidum, salicinum, glucono- $\delta$-lactonum, 2 - et 5 -ketogluconatum nec inositolum.

Kalium nitricum et kalium nitrosum non assimilantur.

Ethyl aminum assimilatur.

Ad crescentiam thiaminum et pyridoxinum necessariae sunt.

Crescere potest in $40-45^{\circ} \mathrm{C}$.

$\mathrm{Mol} \% \mathrm{G}+\mathrm{C}=36.9, \sigma^{2}=0.148$.

Typus: Stirps UCD (FS\&T) 76-243A ex tabidosis sacculis cacti Lemaireocerei thurberi Rancho San Martin, Baja Californiensis isolata est.

Description of $\boldsymbol{P}$. cactophila. Growth in malt extract: After 3 days the cells are ovoid to slightly elongate and measure 1.8 to $5.7 \times 2.9$ to $9.0 \mu \mathrm{m}$. They occur singly, in pairs, and in short chains or small clusters of four to seven cells. Budding occurs on broad shoulders or multilaterally. A finely wrinkled, dull, ascending pellicle is formed, and there is a small amount of sediment.

Growth on malt extract agar: After 3 weeks the streak culture is cream colored, the surface is smooth and dull to semiglossy, the texture is soft, the cross section is flat, and the border is slightly lobed or entire, sometimes showing rudimentary development of pseudomycelium.

Dalmau plate culture on cornmeal agar: Pseudomycelium formation is absent or rudimentary.

Formation of ascospores: The cells are diploid, and most strains produce two hat-shaped spores which are liberated from the ascus at maturity. In rare cases four-spored asci were observed among two-spored asci (Fig. 2). The two-spored strain 17 is homothallic since isolated single spores developed into ascosporulating clones. A few strains produced predominantly four-spored asci, which lyse upon maturity. Strain 118 was shown to be heterothallic by single-spore isolation from four-spored asci. Sporulation is best on YM agar after 5 to 7 days at room temperature.

Fermentation of glucose: Most strains do not produce gas. Others may produce a bubble at 9 days, which may increase to 20 to $40 \%$ of the inverted vial in 21 days.

Assimilation of carbon compounds: Glucose, glucosamine, ethanol, glycerol (latent), DL-lactic 
TABLE 1. Localities and sources of isolates of Pichia cactophila

\begin{tabular}{|c|c|c|c|}
\hline Serial no. of strain & UCD-FST $^{a}$ strain designation & Locality $^{b}$ & Habitat/substrate \\
\hline $1,2,3,4,5$ & $\begin{array}{l}76-204 \mathrm{~A}, 76-205,76-213,76- \\
\quad 215,76-217 \mathrm{~A}\end{array}$ & $\begin{array}{l}\text { Rancho Cuñaño, Baja Calif. Sur, } \\
\text { Mexico (a) }\end{array}$ & $\begin{array}{l}\text { Machaerocereus } \\
\text { gummosus }\end{array}$ \\
\hline 6 & $76-207 \mathrm{~A}$ & $\begin{array}{l}\text { Rancho Cuñaño, Baja Calif. Sur, } \\
\text { Mexico (a) }\end{array}$ & $\begin{array}{l}\text { Lophocereus } \\
\text { schottii }\end{array}$ \\
\hline 7,8 & $76-224 \mathrm{~A}, 76-225$ & $\begin{array}{l}\text { Punta Pescadero, Baja Calif. Sur, } \\
\text { Mexico (b) }\end{array}$ & M. gummosus \\
\hline 9,10 & $76-228 \mathrm{~B}, 76-244 \mathrm{~B}$ & $\begin{array}{l}\text { San Pedro, Baja Calif. Sur, Mex- } \\
\text { ico (c) }\end{array}$ & $\begin{array}{l}\text { Pachycereus prin- } \\
\quad \text { glei }\end{array}$ \\
\hline $\begin{array}{l}11,12,13,14,15 \\
\quad 16\end{array}$ & $\begin{array}{l}\text { 76-230B, 76-232B, 76-233B, } \\
76-236 \mathrm{~B}, \quad 76-238 \mathrm{~B}, \quad 76- \\
241 \mathrm{~B}\end{array}$ & $\begin{array}{l}\text { Todos Santos, Baja Calif. Sur, } \\
\text { Mexico (d) }\end{array}$ & M. gummosus \\
\hline 17 & $76-243 \mathrm{~A}$ (type) & $\begin{array}{l}\text { Rancho San Martin, Baja Calif. } \\
\text { Sur, Mexico (e) }\end{array}$ & $\begin{array}{l}\text { Lemaireocereus } \\
\text { thurberi }\end{array}$ \\
\hline $\begin{array}{l}18,19,20,21,22 \\
23,24,25,26 \\
27,28,29\end{array}$ & $\begin{array}{r}76-246,76-248 \mathrm{~B}, 76-249,76- \\
251,76-252,76-254,76- \\
255,76-257 \mathrm{~A}, 76-258,76- \\
259 \mathrm{~A}, 76-260 \mathrm{~A}, 76-261 \mathrm{~A}\end{array}$ & $\begin{array}{l}\text { Isla San Jose, Baja Calif. Sur, } \\
\text { Mexico (f) }\end{array}$ & M. gummosus \\
\hline $30,31,32$ & $76-265 \mathrm{~A}, 76-266 \mathrm{~B}, 76-267 \mathrm{~B}$ & $\begin{array}{l}\text { Mission San Luis Gonzaga, Baja } \\
\text { Calif. Sur, Mexico (g) }\end{array}$ & M. gummosus \\
\hline 33 & $76-268 B$ & $\begin{array}{l}\text { Mission San Luis Gonzaga, Baja } \\
\text { Calif. Sur, Mexico (g) }\end{array}$ & L. thurberi \\
\hline $34,35,36$ & $76-274 \mathrm{~A}, 76-275 \mathrm{~B}, 76-278 \mathrm{~B}$ & $\begin{array}{l}\text { La Paz, Baja Calif. Sur, Mexico } \\
\text { (h) }\end{array}$ & M. gummosus \\
\hline 37 & $76-276 \mathrm{~A}$ & $\begin{array}{l}\text { La Paz, Baja Calif. Sur, Mexico } \\
\text { (h) }\end{array}$ & L. thurberi \\
\hline $38,39,40,41$ & $\begin{array}{l}\text { 76-284B, 76-292A, 76-293B, } \\
76-294\end{array}$ & $\begin{array}{l}\text { El Centenario, Baja Calif. Sur, } \\
\text { Mexico (i) }\end{array}$ & M. gummosus \\
\hline $42,43,44,45,46$ & $\begin{array}{l}76-298 \mathrm{~B}, 76-299 \mathrm{~B}, 76-300 \mathrm{~B} \\
76-301,76-304 \mathrm{~A}\end{array}$ & $\begin{array}{l}\text { Santiago, Baja Calif. Sur, Mexico } \\
\text { (j) }\end{array}$ & M. gummosus \\
\hline 47 & $76-302$ & $\begin{array}{l}\text { Santiago, Baja Calif. Sur, Mexico } \\
\text { (j) }\end{array}$ & P. pringlei \\
\hline 48 & $76-303 \mathrm{~B}$ & $\begin{array}{l}\text { Santiago, Baja Calif. Sur. Mexico } \\
\text { (j) }\end{array}$ & L. thurberi \\
\hline 49 & $76-3 อ 9 A$ & $\begin{array}{l}\text { Palmilla, Baja Calif. Sur, Mexico } \\
(\mathrm{k})\end{array}$ & M. gummosus \\
\hline $50,51,52$ & $76-318 \mathrm{~B}, 76-319 \mathrm{~B}, 76-321 \mathrm{~A}$ & Loreto, Baja Calif. Sur, Mexico (l) & M. gummosus \\
\hline 53 & $76-322 \mathrm{~A}$ & $\begin{array}{l}\text { Rancho Santa Rosa, Baja Calif. } \\
\text { Sur, Mexico }(\mathrm{m})\end{array}$ & L. thurberi \\
\hline 54 & $76-323 \mathrm{~B}$ & $\begin{array}{l}\text { Rancho Santa Rosa, Baja Calif. } \\
\text { Sur, Mexico (m) }\end{array}$ & M. gummosus \\
\hline 55,56 & $76-324 \mathrm{~A}, 76-325 \mathrm{~A}$ & $\begin{array}{l}\text { Rancho Rosarito, Baja Calif. Sur, } \\
\text { Mexico (n) }\end{array}$ & P.pringlei \\
\hline $57,58,59$ & $76-329 \mathrm{~B}, 76-331 \mathrm{~A}, 76-332$ & $\begin{array}{l}\text { Mulegé, Baja Calif. Sur, Mexico } \\
\text { (o) }\end{array}$ & M. gummosus \\
\hline 60,61 & 76-333A, 76-334A & $\begin{array}{l}\text { Mulegé, Baja Calif. Sur, Mexico } \\
(\text { o })\end{array}$ & P. pringlei \\
\hline 62 & $76-338 \mathrm{~B}$ & $\begin{array}{l}\text { Sierra de San Francisco, Baja } \\
\text { Calif. Sur, Mexico (p) }\end{array}$ & Opuntia sp. \\
\hline 63,64 & $76-342,76-343 B$ & $\begin{array}{l}\text { San Borja, Baja Calif. Norte, } \\
\text { Mexico (q) }\end{array}$ & M. gummosus \\
\hline 65 & $76-345$ & $\begin{array}{l}\text { San Borja, Baja Calif. Norte, } \\
\text { Mexico (q) }\end{array}$ & L. schottii \\
\hline 66 & $76-353 \mathrm{~A}$ & $\begin{array}{l}\text { Las Flores, Bahia de Los Angeles, } \\
\text { Baja Calif. Norte, Mexico (r) }\end{array}$ & M. gummosus \\
\hline 67,68 & $76-358,76-359$ & $\begin{array}{l}\text { Murlegé, Baja Calif. Sur, Mexico } \\
\text { (s) }\end{array}$ & $M$. gummosus \\
\hline 69,70 & $76-366 \mathrm{~A}, 76-367 \mathrm{~B}$ & $\begin{array}{l}\text { San Jose de Castro, Baja Calif. } \\
\text { Sur, Mexico (t) }\end{array}$ & M. gummosus \\
\hline $71,72,73$ & $76-369 \mathrm{~B}, 76-370 \mathrm{C}, 76-371 \mathrm{~B}$ & $\begin{array}{l}\text { San Ignacio, Baja Calif. Sur, Mex- } \\
\text { ico (u) }\end{array}$ & M. gummosus \\
\hline
\end{tabular}


TABLF 1-Continued

\begin{tabular}{|c|c|c|c|}
\hline Serial no. of strain & UCD-FST $^{a}$ strain designation & Locality $^{h}$ & Habitat/substrate \\
\hline $\begin{array}{l}74,75,76,77,78 \\
\quad 79\end{array}$ & $\begin{array}{l}76-373, \quad 76-374 \mathrm{~A}, \quad 76-375 \mathrm{~B}, \\
76-377 \mathrm{~A}, \quad 76-384 \mathrm{~B}, \quad 76- \\
385 \mathrm{~A}\end{array}$ & $\begin{array}{l}\text { Mulegé, Baja Calif. Sur, Mexico } \\
\text { (v) }\end{array}$ & P. pringlei \\
\hline 80 & $76-379 \mathrm{~A}$ & $\begin{array}{l}\text { Mulegé, Baja Calif. Sur, Mexico } \\
\text { (v) }\end{array}$ & L. schottii \\
\hline 81,82 & $76-380 \mathrm{~A}, 76-381 \mathrm{~B}$ & $\begin{array}{l}\text { Mulegé, Baja Calif. Sur, Mexico } \\
\text { (v) }\end{array}$ & M. gummosus \\
\hline 83 & $76-387 \mathrm{~A}$ & $\begin{array}{l}\text { Bahia San Nicolás, Baja Calif. } \\
\text { Sur, Mexico (w) }\end{array}$ & L. schottii \\
\hline 84 & $76-388 \mathrm{~A}$ & $\begin{array}{l}\text { Bahia San Nicolás, Baja Calif. } \\
\text { Sur, Mexico }(w)\end{array}$ & M. gummosus \\
\hline 85 & $76-389 \mathrm{~A}$ & $\begin{array}{l}\text { Isla del Carmen, Baja Calif. Sur, } \\
\text { Mexico (x) }\end{array}$ & L. schottii \\
\hline 86,87 & $77-455,77-456$ & $\begin{array}{l}\text { El Socorro, Baja Calif. Norte, } \\
\text { Mexico (y) }\end{array}$ & M. gummosus \\
\hline $\begin{array}{r}88,89,90,91,92 \\
93,94,95,96 \\
97,98,99,100 \\
101,102,103\end{array}$ & $\begin{array}{l}\text { 77-355A, 77-357B, 77-359A, } \\
77-368 \mathrm{D}, \quad 77-371 \mathrm{C}, \quad 77- \\
376 \mathrm{~A}, 77-378 \mathrm{~A}, \quad 77-379 \mathrm{~A} \\
77-377 \mathrm{~A}, \quad 77-387 \mathrm{~A}, \quad 77- \\
389 \mathrm{~A}, 77-391 \mathrm{~A}, \quad 77-393 \mathrm{~A} \\
\text { 77-396A, 77-400A, 77- } \\
\text { 404A }\end{array}$ & $\begin{array}{l}\text { El Socorro, Baja Calif. Norte, } \\
\text { Mexico (y) }\end{array}$ & $\begin{array}{l}\text { Drosophila moja- } \\
\text { vensis }\end{array}$ \\
\hline 104 & $71-157$ & Alamos, Sonora, Mexico (z) & $\begin{array}{l}\text { Rathbunia ala- } \\
\text { mosensis }\end{array}$ \\
\hline $\begin{array}{r}105, \quad 106, \quad 107 \\
108,109\end{array}$ & $\begin{array}{l}71-158,77-432,77-438,77- \\
\quad 439,77-440\end{array}$ & Navojoa, Sonora, Mexico (aa) & R. alamosensis \\
\hline $\begin{array}{l}110, \quad 111, \quad 112, \\
113, \quad 114, \quad 115\end{array}$ & $\begin{array}{l}77-428,77-430,77-431,77- \\
\quad 434,77-435,77-436\end{array}$ & Navojoa, Sonora, Mexico (aa) & Opuntia sp. \\
\hline 116,117 & $71-159,77-441$ & Navojoa, Sonora, Mexico (aa) & L. thurberi \\
\hline 118 & $77-427$ & Navojoa, Sonora, Mexico (aa) & $\begin{array}{l}\text { Pachycereus pec- } \\
\text { ten-aboriginum }\end{array}$ \\
\hline $119,120,121$ & $76-392 \mathrm{~A}, 76-397 \mathrm{~A}, 76-399 \mathrm{~A}$ & Guaymas, Sonora, Mexico (ab) & L. thurberi \\
\hline 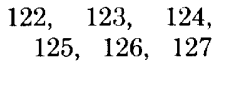 & $\begin{array}{l}76-401 \mathrm{~A}, 76-402 \mathrm{~A}, 76-403 \mathrm{~A} \\
76-404 \mathrm{~A}, \quad 76-405 \mathrm{~A}, \quad 76- \\
406 \mathrm{~A}\end{array}$ & Guaymas, Sonora, Mexico (ab) & R. alamosensis \\
\hline 128 & $76-410 \mathrm{C}$ & Guaymas, Sonora, Mexico (ab) & $P$. pringlei \\
\hline 129 & $71-151$ & $\begin{array}{l}\text { Punta Chueca, Sonora, Mexico } \\
\text { (ac) }\end{array}$ & M. gummosus \\
\hline 130 & $76-500$ & Libertad, Sonora, Mexico (ad) & P. pringlei \\
\hline 131 & $76-502$ & Libertad, Sonora, Mexico (ad) & L. schottii \\
\hline 132 & $71-147$ & Caborca, Sonora, Mexico (ae) & L. thurberi \\
\hline $\begin{array}{c}133, \quad 134, \quad 135 \\
136,37,138\end{array}$ & $\begin{array}{l}71-154,72-308,73-100,73- \\
51,73-57,77-459 B\end{array}$ & Tucson, Ariz. (af) & $\begin{array}{l}\text { Carnegiae gigan- } \\
\text { tea }\end{array}$ \\
\hline $139,140,141,142$ & $\begin{array}{l}72-304,72-324,73-105,77- \\
\quad 461 \mathrm{C}\end{array}$ & Tucson, Ariz. (af) & C. gigantea (soil) \\
\hline 143 & $76-504$ & Tucson, Ariz. (af) & $\begin{array}{l}\text { Ferocactus wisli- } \\
\quad \text { zeni }\end{array}$ \\
\hline $\begin{array}{rr}144, \quad 145, & 146 \\
147, \quad 148, & 149\end{array}$ & $\begin{array}{r}77-462 \mathrm{~A}, 77-463,77-465 \mathrm{~A} \\
77-470 \mathrm{~A}, 77-471,77-478 \mathrm{~A}\end{array}$ & Tucson, Ariz. (af) & $\begin{array}{l}\text { Drosophila ni- } \\
\text { grospiracula fe- } \\
\text { males }\end{array}$ \\
\hline 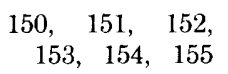 & $\begin{array}{l}77-472,77-473,77-479,77- \\
\quad 484,77-487,77-488\end{array}$ & Tucson, Ariz. (af) & $\begin{array}{l}\text { D. nigrospiracula } \\
\text { males }\end{array}$ \\
\hline $\begin{array}{rr}156, & 157, \quad 158 \\
159, & 160\end{array}$ & $\begin{array}{l}77-466 \mathrm{~A}, 77-467,77-468,77- \\
\quad 496,77-499\end{array}$ & Tucson, Ariz. (af) & $\begin{array}{l}\text { Drosophila } \\
\text { cies "M" spe- } \\
\text { males }\end{array}$ \\
\hline $\begin{array}{lll}161, & 162, & 163, \\
164, & 165, & 166, \\
167, & 168, & 169 \\
170 & & \end{array}$ & $\begin{array}{l}77-464,77-474,77-483 B, 77- \\
485,77-490,77-491,77- \\
494 \mathrm{D}, 77-495,77-498,77- \\
500\end{array}$ & Tucson, Ariz. (af) & $\begin{array}{l}\text { Drosophila spe- } \\
\text { cies "M" males }\end{array}$ \\
\hline 171 & $77-312 B$ & Apatzingan, Michoaćan, Mexico & $\begin{array}{l}\text { Backebergia mili- } \\
\quad \text { taris }\end{array}$ \\
\hline
\end{tabular}


TABLE 1-Continued

\begin{tabular}{|c|c|c|c|}
\hline Serial no. of strain & UCD-FST ${ }^{\prime \prime}$ strain designation & Locality ${ }^{h}$ & Habitat/substrate \\
\hline 172 & $77-314 \mathrm{D}$ & Tlapa, Guerrero, Mexico & $\begin{array}{l}\text { Lemaireocereus } \\
\text { stellatus }\end{array}$ \\
\hline $173,174,175$ & $77-315 \mathrm{~A}, 77-316 \mathrm{~A}, 77-320 \mathrm{~A}$ & Mitla, Oaxaca, Mexico & $\begin{array}{l}\text { Lemaireocereus } \\
\text { pruinosus }\end{array}$ \\
\hline $176,177,178,179$ & $\begin{array}{l}77-317 \mathrm{~A}, 77-318 \mathrm{~A}, 77-323 \mathrm{~A} \\
77-325 \mathrm{~B}\end{array}$ & Mitla, Oaxaca, Mexico & $\begin{array}{l}\text { Lemaireocereus } \\
\text { treleasei }\end{array}$ \\
\hline 180,181 & $77-324 \mathrm{D}, 77-326 \mathrm{~B}$ & Mitla, Oaxaca, Mexico & Myrtillocactus sp. \\
\hline $182,183,184$ & 77-327B, 77-328A, 77-328B & Totolapan, Oaxaca, Mexico & L. pruinosus \\
\hline 185 & $77-329 \mathrm{~A}$ & Totolapan, Oaxaca, Mexico & $\begin{array}{l}\text { Lemaireocereus } \\
\text { dumortieri }\end{array}$ \\
\hline 186 & $77-331 \mathrm{~A}$ & Ixtepec, Oaxaca, Mexico & $\begin{array}{l}P . \text { pecten-abori- } \\
\text { ginum }\end{array}$ \\
\hline $187,188,189$ & 77-332A, 77-333A, 77-334A & Ixtepec, Oaxaca, Mexico & L.pruinosus \\
\hline 190 & $76-900 \mathrm{~B}$ & Saddle Road, Island of Hawaii & $\begin{array}{l}\text { Opuntia mega- } \\
\text { cantha }\end{array}$ \\
\hline
\end{tabular}

${ }^{a}$ UCD-FST, University of California, Davis-Department of Food Science and Technology.

${ }^{b}$ The letters in parentheses correspond to the geographic locations shown in Fig. 1.

acid (latent or weak or negative), succinic acid, and citric acid (rarely negative) are assimilated. The following compounds are not assimilated by any of the strains: D-galactose, $\mathrm{L}$-sorbose, maltose, sucrose, cellobiose, trehalose, lactose, melibiose, raffinose, melezitose, inulin, soluble starch, D-xylose, L-arabinose, D-arabinose, D-ribose, L-rhamnose, methanol, erythritol, ribitol, galactitol, D-mannitol, D-glucitol, $\alpha$-methyl-Dglucoside, salicin, glucono- $\delta$-lactone, gluconic acid, 2-keto- and 5-ketogluconic acid, and mesoinositol.

Assimilation of nitrogen compounds: Potassium nitrate, -; potassium nitrite, -; ethylamine, + ; ammonium sulfate, + .

Growth in vitamin-free medium: Negative. Thiamine and pyridoxine are essential for growth.

Growth on $50 \%$ (wt/wt) glucose-yeast extract agar: Negative.

Growth on $10 \% \mathrm{NaCl}$ plus $5 \%$ glucose in yeast autolysate agar: Variable. Some strains show no growth; others grow moderately.

Maximum temperature for growth: Nearly all strains grow well at $42^{\circ} \mathrm{C}$. Some grow moderately at $45^{\circ} \mathrm{C}$.

Acid formation on chalk agar: Weak to moderate or absent.

Hydrolysis of urea: Negative.

Synthesis of starchlike compounds: Negative.

Gelatin liquefaction: Negative.

Casein hydrolysis: Negative.

Lipolytic activity: Negative.

Growth in the presence of $0.1 \mathrm{mg}$ of cycloheximide per ml: Negative.

$\mathrm{G}+\mathrm{C}$ content of the nuclear DNA: 36.9 $\mathrm{mol} \%, \sigma^{2}=0.148$ (based on 9 strains [Table 2]).

Habitat: One hundred ninety strains were isolated during the period 1971 to 1976 from a wide variety of cactus species and over a wide geo- graphic area (see Fig. 1 and Tables 1 and 3).

Type: The type strain, UCD-FS\&T 76-243A, isolated from necrotic tissue of Lemaireocereus thurberi (organ pipe cactus) near Rancho San Martin, Baja California Sur, Mexico, has been deposited in the collection of the Yeast Division of the Centraalbureau voor Schimmelcultures in Delft, The Netherlands, as CBS 6926 and in the American Type Culture Collection, Rockville, Md., as ATCC 34932.

\section{DISCUSSION}

Among the new Pichia species that we have recognized in cactus rots, $P$. cactophila shows the widest distribution, both in terms of geography and host-plant specificity (Table 3). It was isolated from all species of cactus throughout the entire North American Sonoran Desert. Some isolates were recovered from cacti or drosophilae outside the Sonoran Desert, namely, strain 190 from Opuntia megacantha on the island of Hawaii and strains 171-189 from various cacti growing in southern Mexico; one strain (Table 2) had been isolated in 1950 from the crop of Drosophila pseudoobscura Frolowa at Piñon Flat (southern California) by Shehata (A. M. El-Tabey Shehata, Ph.D. thesis, University of California, Berkeley, 1950). This last strain was initially considered as belonging to a new species of Pichia by Shehata, but it was later regarded as a strain of $P$. membranaefaciens (12).

The metabolic capability of $P$. cactophila, like that of $P$. membranaefaciens, is limited to the oxidative utilization of only a few compounds (see standard description of $P$. cactophila). The only phenotypic property that distinguishes the two species is the strong growth exhibited by $P$. cactophila on D-glucosamine, a compound not 


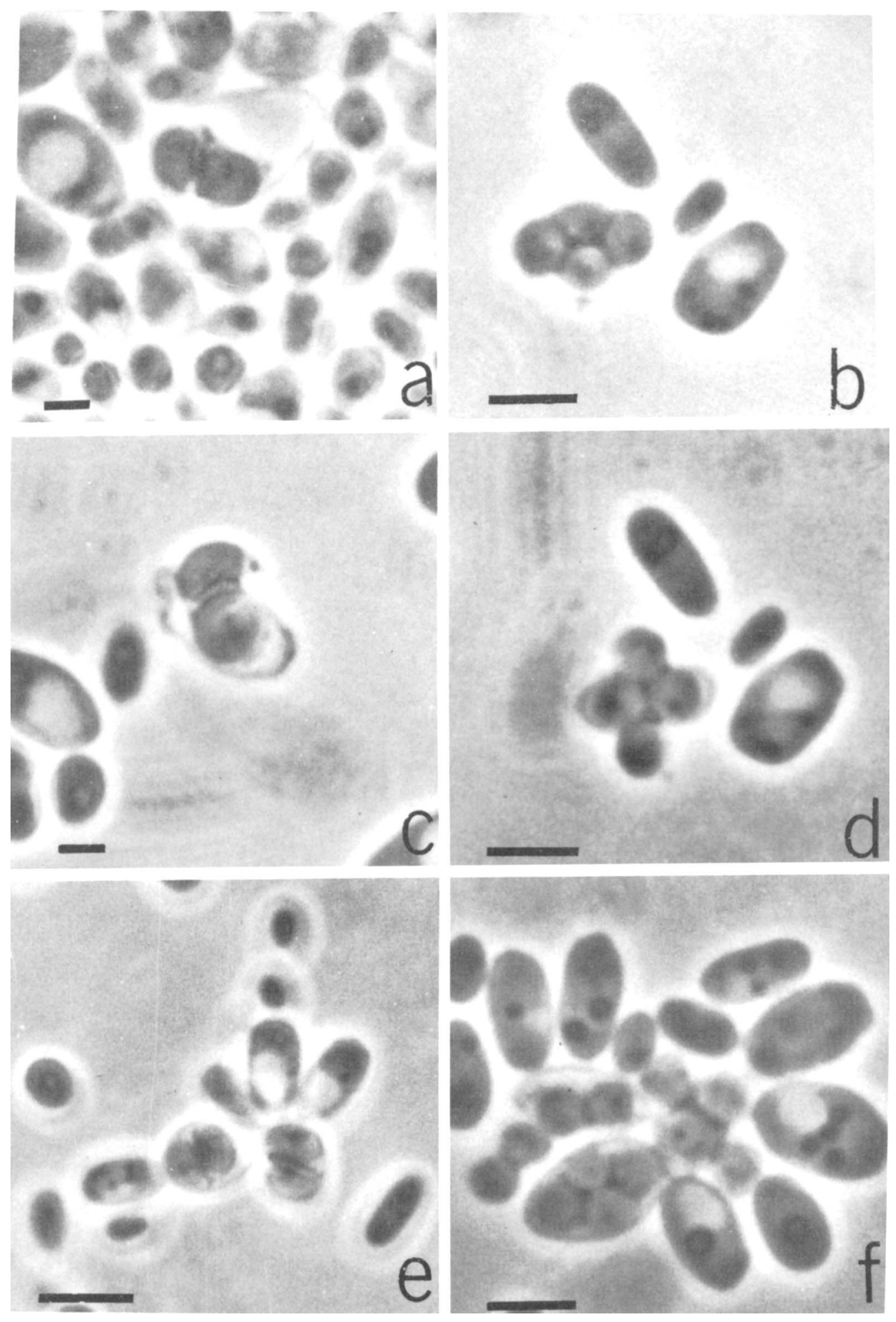

FIG. 2. Intact asci of Pichia cactophila strain 17 (a) and the four-spored strain 118 (b) have thin walls. As the thin ascus wall lyses (c), the brim of the spore becomes more evident. In the four-spored strain, the dehisced ascospores appear to remain physically associated (d and f) and form clumps. Each spore contains a highly refractile body (strain 94 [e]). The sporulation cycle is completed within $24 \mathrm{~h}$ or less. Bars represent $2 \mu \mathrm{m}$ in all cases. 
TABLE 2. Nuclear DNA base compositions of representative strains of $P$. cactophila

\begin{tabular}{|c|c|c|c|c|}
\hline Strain & Locality & Substrate & $\begin{array}{l}\mathrm{G}+\mathrm{C} \text { con- } \\
\text { tent (mol\% } \pm \\
\mathrm{SD})^{\prime} \text { of nu- } \\
\text { clear DNA }\end{array}$ & $\begin{array}{l}\text { Predomi- } \\
\text { nant no. } \\
\text { of } \\
\text { spores/ } \\
\text { ascus }\end{array}$ \\
\hline 17 (type) & $\begin{array}{l}\text { Rancho San Martin, Baja Calif., } \\
\text { Mexico }\end{array}$ & Lemaireocereus thurberi & $36.3 \pm 0.27$ & 2 \\
\hline 52 & Loreto, Baja Calif., Mexico & Machaerocereus gummosus & $36.5 \pm 0.14$ & 2 \\
\hline 69 & $\begin{array}{l}\text { San Jose de Castro, Baja Calif., } \\
\text { Mexico }\end{array}$ & M. gummosus & $36.7 \pm 0.13$ & 2 \\
\hline 118 & Navojoa, Sonora, Mexico & $\begin{array}{l}\text { Pachycereus pecten-abori- } \\
\text { ginum }\end{array}$ & $37.1 \pm 0.05$ & 4 \\
\hline 171 & Apatzingan, Michoacán, Mexico & Backebergia militaris & $36.9 \pm 0.11$ & 4 \\
\hline 175 & Mitla, Oaxaca, Mexico & Lemaireocereus pruinosus & $37.3 \pm 0.07$ & 2 \\
\hline 182 & Totolapan, Oaxaca, Mexico & L. pruinosus & $37.5 \pm 0.45$ & 4 \\
\hline 190 & Island of Hawaii & Opuntia megacantha & $37.1 \pm 0.23$ & 2 \\
\hline $50-44^{h}$ & Piñon Flat, Calif. & $\begin{array}{l}\text { Drosophila pseudoobscura } \\
\text { Frolowa }\end{array}$ & $37.1 \pm 0.36$ & 2 \\
\hline
\end{tabular}

" $\mathrm{G}+\mathrm{C}$ values for strains 17 and 52 were based on three separate determinations; two separate determinations were utilized for all other strains. SD, Standard deviation.

${ }^{h}$ Isolated by El-Tabey Shehata in 1950.

TABLE 3. Sources and frequencies of isolation of strains of Pichia cactophila

\begin{tabular}{|c|c|}
\hline Source & $\begin{array}{l}\text { Frequency of } \\
\text { isolation }\end{array}$ \\
\hline $\begin{array}{c}\text { Machaerocereus gummosus Engelmann } \\
{\text { [Agria }]^{n}}^{n}\end{array}$ & $64^{b}$ \\
\hline $\begin{array}{l}\text { Lemaireocereus thurberi Engelm. [Or- } \\
\text { ganpipe] }\end{array}$ & 11 \\
\hline $\begin{array}{l}\text { Lemaireocereus pruinosus (Otto) Brit- } \\
\text { ton and Rose }\end{array}$ & 9 \\
\hline $\begin{array}{l}\text { Lemaireocereus treleasei Britt. and } \\
\text { Rose }\end{array}$ & 4 \\
\hline $\begin{array}{c}\text { Lemaireocereus stellatus (Pfeiffer) } \\
\text { Britt. and Rose }\end{array}$ & 1 \\
\hline $\begin{array}{l}\text { Lemaireocereus dumortieri (Scheid- } \\
\text { weiler) Britt. and Rose } \ldots \ldots \ldots \ldots\end{array}$ & 1 \\
\hline $\begin{array}{l}\text { Pachycereus pringlei (S. Wats.) Britt. } \\
\text { and Rose [Cardon] } \ldots \ldots \ldots \ldots \ldots\end{array}$ & 15 \\
\hline $\begin{array}{l}\text { Pachycereus pecten-aboriginum (En- } \\
\text { gelm.) Britt. and Rose [Hecho] } \ldots . .\end{array}$ & 2 \\
\hline Carnegiae gigantea Engelm. [Saguaro] & 6 \\
\hline C. gigantea soil $\ldots \ldots \ldots \ldots \ldots$ & 4 \\
\hline Lophocereus schottii Engelm. [Senita] & 6 \\
\hline $\begin{array}{l}\text { Rathbunia alamosensis (Coulter) Britt. } \\
\text { and Rose [Cina] } \ldots \ldots \ldots \ldots\end{array}$ & 12 \\
\hline $\begin{array}{l}\text { Ferocactus wislizeni (Engelm.) Britt. } \\
\text { and Rose [Barrel] } \ldots \ldots\end{array}$ & 1 \\
\hline Myrtillo cactus sp. . . . & 2 \\
\hline $\begin{array}{l}\text { Backebergia militaris (Audot) Bravo } \\
\text { and Sanchez-Mejorada }\end{array}$ & 1 \\
\hline Opuntia megacantha Salm-Dyck ..... & 1 \\
\hline Opuntia sp. $\ldots \ldots \ldots \ldots \ldots \ldots \ldots$ & 7 \\
\hline $\begin{array}{c}\text { Drosophila nigrospiracula Patterson } \\
\text { and Wheeler }\end{array}$ & 12 \\
\hline $\begin{array}{l}\text { Drosophila mojavensis Patterson and } \\
\quad \text { Crow }\end{array}$ & 16 \\
\hline Drosophila species "M" & 15 \\
\hline
\end{tabular}

${ }^{a}$ Common plant names are in brackets.

${ }^{b}$ Number of times $P$. cactophila was isolated from source. routinely employed by most yeast taxonomists. This limited set of utilizable compounds suggests that $P$. cactophila probably invades the decaying cactus tissue after active fermentation by other microorganisms has begun. Species in the bacterial genus Erwinia are thought to initiate the necrosis and render the succulent tissue vulnerable to saprophytic yeasts (6). Presumably adult drosophilae serve as vectors for dispersal of $P$. cactophila (as well as other yeast species) since the yeast is readily recovered from the adult crops (Table 1). The yeast-laden cactus tissue is then utilized by cactophilic drosophilae for feeding and breeding. The adaptation of $P$. cactophila to desert habitats is reflected in its relatively high maximum temperature for growth $\left(40\right.$ to $\left.45^{\circ} \mathrm{C}\right)$.

$P$. cactophila is distinctly different from $P$. membranaefaciens in DNA base composition: the former shows a range of 36.3 to $37.5 \mathrm{~mol} \%$ and the latter has a range of 42 to $44 \mathrm{~mol} \% \mathrm{G}+\mathrm{C}$ $(8,9)$. In addition to its ability to utilize $D$ glucosamine, the cactus habitat of $P$. cactophila is helpful in separating the physiologically similar $P$. membranaefaciens. Although $P$. cacto. phila had been regarded as a strain of P. membranaefaciens $(4,14)$, the present taxonomic study indicates that $P$. membranaefaciens is not associated with necrotic cactus tissue. A parallel ecological situation exists for certain isolates of $P$. membranaefaciens. This species (also referred to as $P$. silvestris, later regarded as synonymous with $P$. membranaefaciens [5]) is known to occur in slime fluxes of temperate trees (10), and it has been isolated from tree fluxes in which immature stages of drosophilae were found $(2,4)$. The similarity in ecology and phys- 
iology of $P$. membranaefaciens and $P$. cacto. phila suggests that they are closely related, but the wide differences in their DNA base compositions indicates the possibility of evolutionary convergence. This in large part depends on the proximal causes of DNA changes before and after yeast "speciation." A better understanding of the relationship of the two taxa might be established through comparative studies of yeasts found in tree fluxes of desert and tropical regions, especially in localities where trees and cacti are known to have been sympatric for some time. A comparison of molecular changes that could establish evolutionary distance (e.g., micro-complement fixation and nucleic acid base sequence comparison) would also be helpful in elucidating the evolutionary relationships of $P i$. chia species inhabiting tree fluxes and cactus rots. Phylogenetic considerations remain speculative, but ecological knowledge combined with modern biochemical techniques could provide promising data for future studies in yeast phylogeny. A comparison of $\mathrm{G}+\mathrm{C}$ values for strains of $P$. cactophila isolated from the Sonoran Desert (strains 14, 52, 69, and 118) with those found outside this region (strains 171, 175, 182, 190, and 50-44) shows that the two distinct geographic groups exhibit significantly different means ( $t=2.53, \alpha=0.05)$. The mean $\mathrm{G}+\mathrm{C}$ value of strains outside of the Sonoran Desert is higher than the mean of strains within the desert by $0.51 \mathrm{~mol} \%$. We are continuing to explore the evolutionary significance of this difference. The large number of isolates and the widely separated geographic localities of isolation (Table 1 and Fig. 1) provide valuable material for intraspecific population studies. Very few eucaryotic microorganisms have been investigated for allozyme variation (see Spieth [13] for an analysis of Neurospora intermedia). Although most strains of $P$. cactophila are assumed to be homothallic, a study of conspecific populations for variability of isozymes and inferred allozyme variation is likely to be rewarding.

\section{ACKNOWLEDGMENTS}

We are greatly indebted to Nancy Krauter for her help in determining the DNA base compositions and to André Lachance for the preparation of the illustrations and for his assistance with the Latin diagnosis. We greatly appreciate assistance with the field collections by Alexander Russell, Jr., Jean S. Russell, William R. Johnson, Don C. Vacek, and Robert L. Mangan. A gift of zymolyase from the Research Laboratories of Kirin Breweries Ltd., Takasaki, Japan, is gratefully acknowledged.

This research was supported by Public Health Service grant GM-16307-07 from the National Institute of General Medical Sciences to $\mathrm{H}$. J. Phaff and by a grant from the
National Science Foundation to W. B. Heed and H. W. Kircher (DEB 74-19148A03).

\section{REPRINT REQUESTS}

Address reprint requests to: $\mathrm{H}$. J. Phaff, Department of Food Science and Technology, University of California, Davis, CA 95616 .

\section{LITERATURE CITED}

1. Bernardi, G., M. Foures, G. Piperno, and P. P. Slonimski. 1970. Mitochondrial DNAs from respiratory-sufficient and cytoplasmic respiratory-deficient mutants of yeast. J. Mol. Biol. 48:23-42.

2. Carson, H. L., E. P. Knapp, and H. J. Phaff. 1956. Studies on the ecology of Drosophila in the Yosemite region of California. III. The yeast flora of the natural breeding sites of some species of Drosophila. Ecology 37:538-544.

3. Fowell, R. R. 1969. Sporulation and hybridization of yeasts, p. 363 . In A. H. Rose and J. S. Harrison (ed.), The yeasts, vol. 1. Academic Press Inc., New York.

4. Heed, W. B., W. T. Starmer, M. Miranda, M. W. Miller, and H. J. Phaff. 1976. An analysis of the yeast flora associated with cactiphilic Drosophila and their host plants in the Sonoran Desert and its relation to temperate and tropical associations. Ecology 57:151-160.

5. Kreger-van Rij, N. J. W. 1970. Pichia Hansen, p. 455-554. In J. Lodder (ed.), The yeasts-a taxonomic study. North-Holland Publishing Co., Amsterdam.

6. Lightle, P. C., E. T. Standring, and J. G. Brown. 1942. A bacterial necrosis of the giant cactus. Phytopathology 32:303-313.

7. Marmur, J. 1961. A procedure for the isolation of DNA from microorganisms. J. Mol. Biol. 3:208-218.

8. Meyer, S. A., and H. J. Phaff. 1970. Taxonomic significance of the DNA base composition in yeast, p. 1-29. In D. G. Ahearn (ed.), Recent trends in yeast research, Spectrum vol. 1. Georgia State University, Atlanta.

9. Nakase, T., and K. Komagata. 1970. Significance of DNA base composition in the classification of the yeast genus Pichia. J. Gen. Appl. Microbiol. 16:511-521.

10. Phaff, H. J., and E. P. Knapp. 1956. The taxonomy of yeasts found in exudates of certain trees and other natural breeding sites of some species of Drosophila. Antonie van Leeuwenhoek J. Microbiol. Serol. 22:117-130.

11. Schildkraut, C. L., J. Marmur, and P. Doty. 1962. Determination of the base composition of deoxyribonucleic acid from its buoyant density in $\mathrm{CsCl}$. J. Mol. Biol. 4:430-433.

12. Shehata, A. M. El Tabey, E. M. Mrak, and H. J. Phaff. 1955. Yeasts isolated from Drosophila and from their suspected feeding places in southern and central California. Mycologia 47:799-811.

13. Spieth, P. T. 1975 . Population genetics of allozyme variation in Neurospora intermedia. Genetics 80:785-805.

14. Starmer, W. T., W. B. Heed, M. Miranda, M. W. Miller, and H. J. Phaff. 1976. The ecology of yeast flora associated with cactiphilic Drosophila and their host plants in the Sonoran Desert. Microb. Ecol. 3:11-30.

15. Szybalski, W. 1968 . Use of cesium sulfate for equilibrium density gradient centrifugation. Methods Enzymol. 12B:330-360.

16. Van der Walt, J. P. 1970. Criteria and methods used in classification, p. 34-113. In J. Lodder (ed.), The yeasts-a taxonomic study. North-Holland Publishing Co., Amsterdam. 